\title{
Impacts of Radio-Identification on Cryo-Conservation Centers
}

\author{
Sylvain HOUSSEMAN \\ Nabil ABSI \\ Stéphane DAUZERE-PERES \\ Dominique FEILLET
}

\author{
Ecole des Mines de Saint-Etienne \\ CMP - Site Georges Charpak 880, Avenue de Mimet \\ F-13541 Gardanne France
}

\section{November 2009 \\ Working paper ENSM-SE CMP WP 2009/15}

This paper deals with using simulation as a decision support tool for estimating the impact of Radio Frequency IDentication (RFID) technologies within biological sample storage areas (called biobanks). Several indicators, including inventory reliability or human resource utilization, are compared and discussed for different scenarios of use of the technologies. A special emphasis is put on the so-called re-warehousing activity, which RFID makes possible and consists in reassigning tubes to empty sockets when boxes are emptied. For this particular activity, optimization algorithms are developed and embedded in the simulator. Results demonstrate the potential interest of RFID in biobanks and the value of simulation for estimating and optimizing such complex socio-technical systems. 
pdfauthor

\title{
IMPACTS OF RADIO-IDENTIFICATION ON CRYO-CONSERVATION CENTERS
}

\author{
Sylvain Housseman, Nabil Absi, \\ Dominique Feillet and \\ Stéphane Dauzère-Pérès \\ Ecole des Mines de Saint-Etienne \\ CMP Georges Charpak \\ F-13541, Gardanne, France. \\ [housseman, absi, feillet, dauzere-peres]_at_emse.fr
}

This paper deals with using simulation as a decision support tool for estimating the impact of Radio Frequency IDentification (RFID) technologies within biological sample storage areas (called biobanks). Several indicators, including inventory reliability or human resource utilization, are compared and discussed for different scenarios of use of the technologies. A special emphasis is put on the so-called re-warehousing activity, that RFID makes possible and which consists in reassigning tubes to empty places when boxes are emptied. For this particular activity, optimization algorithms are developed and embedded in the simulator. Results demonstrate the potential interest of RFID in biobanks and the value of simulation for estimating and optimizing such complex socio-technical systems.

Categories and Subject Descriptors: L.N1.N2 [Category]: Category and Categoriz-documentation; L.N1.N2 [Information Systems Applications]: General; I.7.2 [Text Processing]: Document Preparation-languages; photocomposition

General Terms: Documentation, Languages

Additional Key Words and Phrases: RFID, Healthcare, Simulation, Biobanks

\section{INTRODUCTION}

Trying to estimate the impact of introducing auto-IDentification technologies in a logistic process is a complex topic. The possibilities offered by these technologies may indeed affect the process in many different ways, at several decision levels, sometimes even allowing for drastic changes. Simulation offers a practical tool for addressing these questions without referring to costly and very focused pilot studies. This paper proposes to use simulation to estimate the impact of introducing Radio Frequency IDentification (RFID) technologies within biobanks. This study is part of the so-called MISTRALS project, involving industrial companies (Tagsys, IBM, Psion Teklogics and SPS); two French health structures developing a biobank activity: the Institute Paoli-Calmettes (IPC), in Marseilles, and the teaching hospital of Nice (CHU-N); and two research departments of the École Nationale Supérieure des Mines de Saint-Étienne.

Pilot plants and demonstrators, which can be considered as real-life simulators, are certainly the best way to assess the behavior of a contactless communication system within a particular environment, even though they are expensive, potentially risky and hardly extensible [Royston 1999]. Furthermore, they are able to provide very precise information on the system (e.g., times captured by RFID) that can help defining acute parameters for simulating relatively similar processes [Boginski et al. 2007]. In the case of the MISTRALS project, the development of a demonstrator is scheduled. Unfortunately, at this stage of the 
project, the demonstrator is still not available. As a consequence, the use case presented in this paper is based on forecasted and estimated values.

\subsection{What is Radio IDentification}

RFID is the generic acronym for Radio Frequency IDentification (or Radio Frequency Identification Devices). Smart objects are assets that are tagged with an RFID transponder (or tag), and thus can transfer and record information without any human action. Inductively coupled RFID systems work as follows: a base-station antenna sends electromagnetic waves that are captured by the RFID tag's antenna. These waves allow empowering the tag's chip, which modifies the signal. The reader's antenna decrypts the modifications and thus deduces the information embedded in the tag [Hedgepeth 2007]. Depending on the technology, different frequency bands, ranging from Low Frequency $(125 \mathrm{kHz})$ to Super High Frequencies $(5.8 \mathrm{GHz})$, can be used, with different data transfer rates and reading capabilities. New generations of RFID tags, readers and protocols allow for large amount of data (a few kilobytes), multiple reading and writing, on-chip sensors to be added [Miles et al. 2008] and allow objects to communicate with each other. The use of RFID is growing fast. IDTechEx [IDTechEx 2009] estimates that 2.35 billion tags will be sold in 2009, versus 1.97 billion in 2008, 1.74 billion in 2006 and 1.02 billion in 2005. The appearance of standards and protocols, the improvement of the technologies and manufacturing techniques and the large spectrum of applications in many different domains boost demands, thus reducing price of RFID technologies.

\subsection{Uses and impact studies of RFID}

A prominent domain of application for RFID technologies is supply chain management. Applications are numerous including simple display of information, recording of statistics for planning and scheduling, traceability, inventory management, order verification and quality control. RFID is however applied to many other domains and new applications appear at a very high rate, notably in healthcare contexts. The pressure for a better traceability in this domain (though already of obvious importance) is growing fast for several reasons: new legislations in France force a new drug pedigree using at least DataMatrix codes, the Activity Based Costing (ABC) principle starts being effective in French public hospitals (FHF, 2008), the USA are facing their third malpractice crisis, physicians struggling to acquire malpractice affordable insurances [Dalton et al. 2008] while a better traceability could reduce medical errors and improve the tracking of their causes. In this context, crossing the technological pitch of using smart objects is clearly an issue. In 2008, 10 millions of tags were used for drugs, and 15 millions for other healthcare applications. Many obvious possibilities have already been addressed like: automatic verification of the compliance to prescription, automatic checking of use-by dates or sterilization processing, detection, management and sharing of devices. The database of IDTechEx contained 217 use cases in October 2009. Recently RFID technology was adapted allowing tags to resist gamma ray sterilization [Andrechak and Wiens 2006], which may allow more applications, like tagging standard cryotubes and re-sterilizing them afterwards. One should also mention and insist on the role that RFID could play against drug counterfeit. The medical area accuses a high counterfeit proportion, attaining the alarming rate of $70 \%$ in certain regions of the world, while the overall proportion could be more than $10 \%$, representing a market supposed to attain 75 billion USD in 2010 [WorldHealthOrganization 2006] [IMPACT 2006]. RFID tagging is advanced as a solution to avoid or significantly reduce "grey mar- 
ket" and counterfeit reselling, notably compared with the different kinds of data-matrices.

Many studies try to estimate the impacts of RFID technologies in retail stores and supply chains, including the possibility of reducing the stocks (thanks to inventory accuracy in real time [de Kok et al. 2007]), the bullwhip effect or shoplifting [Rekik 2006] [Sarac et al. 2008]. Some studies are based on analytical models while others use simulation. One of the main difficulties is due to the complexity of the systems, the presence of unpredictable events and the many device and organizational configurations. Other important difficulties, raised for example by [Gaukler et al. 2007], are that some parts of the systems can be used for other applications, that information can be beneficial for different stakeholders and that the costs should be shared in consequences.

Finally, we can read in a recent paper [Lin 2009] that "the efforts for a complete adoption of RFID technology are usually underestimated and the real benefits are optimistically overestimated"; this is in this context that we propose a re-configurable simulation model that aims at estimating the impacts of implementing RFID within a biobank.

\subsection{RFID and biobanks}

Biobanks, or more generally Biological Resource Centers, have to operate the tasks of receiving, transforming, storing and making available biological (human) samples and their derivatives. The biobanks considered in this study stock samples at very low temperatures $\left(-196{ }^{\circ} \mathrm{C}\right)$, in nitrogen tanks. Samples are intended to be used within research programs and for diagnosing or evaluating the risk to develop cancer. Biobank activities can be regarded as intermediate between stock management and health delivery. Like most of the health production systems, many human manipulations happen. Traceability is undoubtedly of importance for biobanks and smart objects are natural candidates for contributing to improvements in this direction. Those observations make the biobank case interesting as it reflects the possibilities RFID can bring in more general contexts.

The RFID technology we want to test needs tagged cryotubes to be read individually, without endangering the patient's privacy and with the possibility to read and write data. The cryotubes will be put into boxes, themselves stocked in nitrogen tanks. Low-range (High Frequency, $13.56 \mathrm{MHz}$ ) technology was chosen as a prerequisite. There are no a priori proofs that the information written on the tags will be kept if stocked for years in a nitrogen tank. [Bettendorf et al. 2008] however show that an RFID tag stocked at a very low temperature for 18 months can still be read. Accelerated physical and thermal aging tests [Cauchois 2008] are currently being conducted at the École des Mines de Saint-Étienne. Tags are attached to cryotubes and tested conserved for the equivalent of 10 year storage at a very low temperature. The tests include reading tests, where it has been observed that the tags can be read a few seconds after they are pulled out from the nitrogen freezers, their awaking temperature being $-158.5^{\circ} \mathrm{C}$.

Another important issue is the impact of electromagnetic interferences on medical machines and human cells. [Togt et al. 2008] point that incompatibility problems can make some material configurations impossible. [Hohberger and Tsirline 2009] show that long term exposure of red blood cells and platelets to the 13,56MHz RFID technology has no adverse impact. [Arumugam and Engels 2009] conducted tests showing that the absorption rate of a Ultra High Frequency RFID at $10 \mathrm{~cm}$ is above the allowed limits for safe exposure to RF radiation in public as allowed by the Federal Communications Commission. Wireless technologies still lever ethical and health related questions, but the application of 
HF-RFID in biobanks is technically conceivable.

In biobanks, one can see at least two possible advantages of implementing RFID. First, automatic, acute and ubiquitous traceability of initial and finished products, devices, and mayhap persons could help reducing the number of miss-identification incidents and retrieval problems. Secondly, RFID could allow a better use of the storage area. In many biobanks (and true for the two biobanks involved in the project), sockets left empty because of handover requests are not reused and many boxes of cryotubes in nitrogen tanks tend to contain more and more empty sockets. It is indeed considered that re-warehousing is too risky as it might expose samples to repetitive and uncontrolled changes of temperature. RFID should support this process. This possibility is all the more interesting given that the activity of biobanks seems bound to increase in the next few years. The number of cancers is indeed growing as the population is getting older and because some recent technologies and habits may be triggering factors. Also, the number of handover requests may increase because of new techniques like toxicogenomics, the use of progenitor cells and cord-blood, or the appearance of on-line sample catalogs [e cancer.fr 2007].

The number of possible device configurations and resulting organizational scenarios is large, thus justifying the use of simulation for testing some of them. The dynamic aspect of health production systems, its importance in the appearance of errors, as pictured by the "Swiss cheese model" of system failures [Reason ], and the fact that RFID technologies can bring up new activities, are other reasons that led us to use an approach based on discrete event dynamic simulation (DEDS). The virtual system can thus include the appearance of incidents and a possibility for them to be rectified before accidents happen.

The originality of this study lies in:

- the studied production system, a complex socio-technical system where errors may occur,

-its modeling permitting the implementation of different material and organizational scenarios including smart objects,

— the study of a new activity, namely re-warehousing, allowed by RFID,

-the combination of optimization and simulation for scenarios including re-warehousing activity.

This article is decomposed as follows: Section 2 describes precisely biobank processes, insisting on the possible malfunctions and some RFID-related forecasted improvements, including the description of the re-warehousing activity. Section 3 presents the simulation approach for this study, describes the tested scenarios and the optimization algorithm for the new activity, and presents the considered performance indicators. Section 4 describes our computational experiments, presents the parameters of the simulation and dicusses the results. The last section is a conclusion leveraging future research perspectives.

\section{BIOBANK FLOW DESCRIPTION AND RFID RELATED IMPROVEMENTS}

In this section we describe the mains steps undergone by a biological sample in biobanks, from the sampling to the shipment to final users. This description is generic, it includes

ACM: Transactions on Modeling and Computer Simulation, Vol. ?, No. ?, ?? 20?? 
transformation protocols (like DNA extraction), transitory and final storages. These processes were defined in common with the IPC and the CHU-N. In these processes, paper sheets are associated with samples. Besides this description, we emphasize the problems that might occur during a sample processing and the potential improvements that could be achieved when introducing RFID technologies.

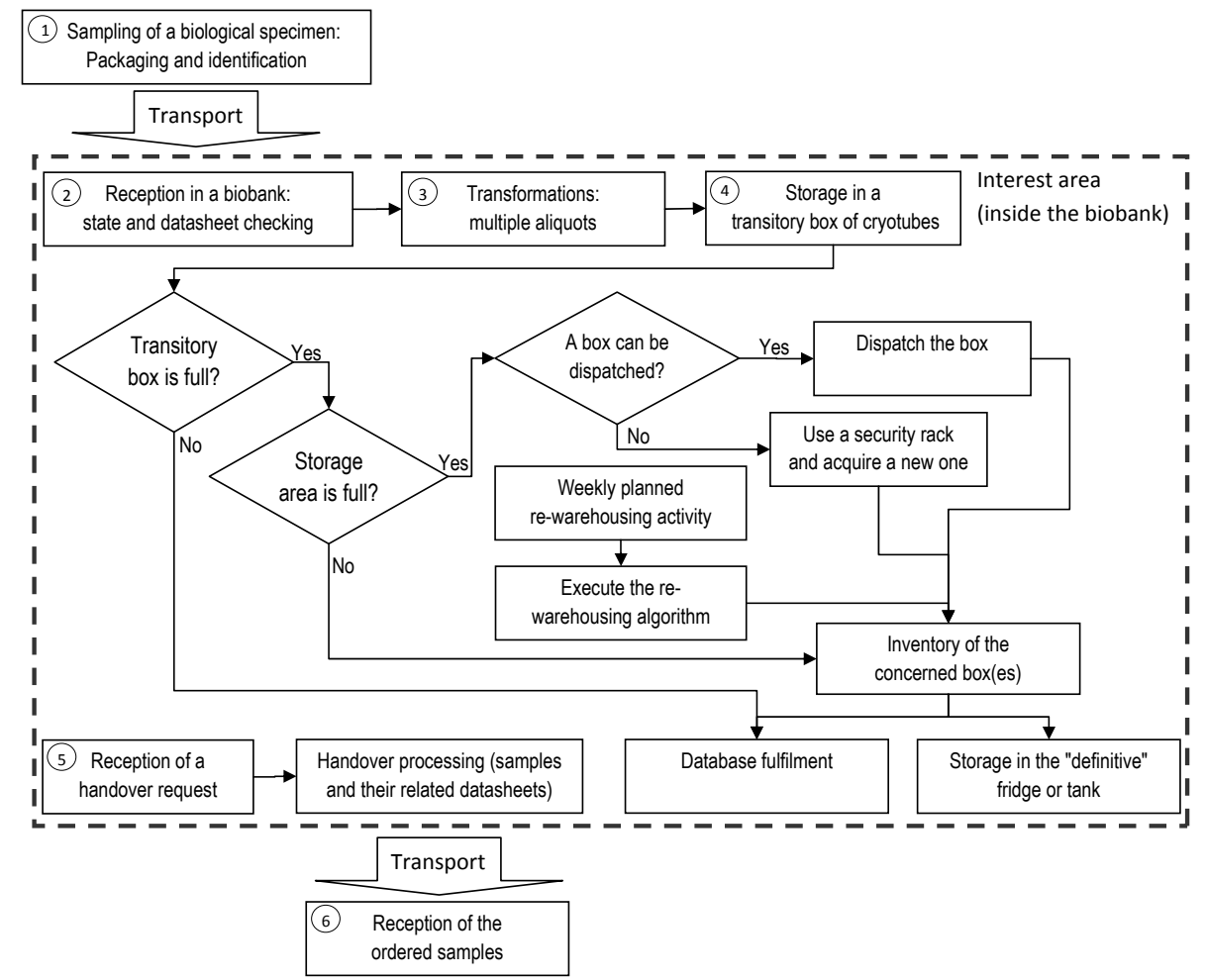

Fig. 1: Biobanks processes diagram

The main advantages for using RFID technologies instead of bar-codes are: no need of direct line of sight, simultaneous data capture, data storage potential, longer distance of reading, and a possible resistance to harsh environments. These two last points are controversial since some environments (metal, water) could prevent radio communication; for these cases special tags and packagings are required, and the reading distance highly depends on the technology and devices that are used.

Six main steps were identified during a sample life as depicted in Figure 1. The first one is the act of sampling (1), including the preparation of needed tools and containers. It does not necessary take place inside the biobanks but samples have to be linked to patient identity. The first process inside the biobank is the reception of the original sample (2). This sample is transformed (3), cut out or separated into multiple samples called aliquots in the sequel. Aliquots are conserved (4) generally at a very low temperature, and sometimes inside paraffin or formalin, depending on the type of sample. The next step is 
the processing of a handover request (5) that can be dedicated to a diagnosis process or a research experiment. The last process (6) is the reception of the requested aliquots by the end-user. These aliquots must be linked to the patient's information but not his identity.

In the following subsection, we jointly describe in detail all the processes and the impacts that RFID could have at the operational level. The new activity of re-warehousing allowed whith RFID technologies will be described in Subsection 2.2.

\subsection{Detailed description of the processes}

The transportation of a biological sample (Step 1) must observe specific conditions regarding transportation time, packaging and temperature. This sample can arrive through a pneumatic transportation device or be carried by a human. The current information system does not allow important information to be retrieved, such as transportation duration, physical shocks, and cold chain failure. The data associated with the sample are also generally handwritten and are sometimes received after the biological piece, which can lead to identification errors. RFID technologies can secure this process. They allow capturing the temperature from a sensor directly linked to the sample during the transportation process. The automatic read/write function allows the traceability of the sample and then reproducing the transportation conditions (temperature, shocks, duration, etc.). A common database update allows information related to the sample to be modified even if it has already been sent.

When a sample is received in the biobank (Step 2) a storage ID is assigned and its state and associated data are checked. Then, the required protocols and transformations into aliquots are performed by anatomopathologist physicians and technicians (Step 3). The transformation protocols retained for modeling are DNA and RNA extractions, white-cells (that are also part of the previous ones), blood serum, plasma, and viable cells. Some of these protocols are different whether the arriving sample is blood or marrow. Transformations may take several days (e.g., in case of cellular culture) which may create identification errors, especially as the manipulator may change. One of the only ways for differentiating pathological and healthy aliquots is based on the color of the tube caps. We note that tube caps could be inverted, and then create diagnosis errors that could have been avoided by saving the data directly on a transponder or in the linked database.

Once transformed and correctly packaged, the sample's aliquots are stored (Step 4). In this study, we only consider the very low temperature conservation case. The packages generally used are cryotubes, stocked in boxes of variable capacity. For the time being, technicians manually input the data related to the sample and its aliquots into the information system (IS), which is also an error prone process. Automatic information transfer using auto-ID technologies could reduce errors. At every check point, data could be sent to the IS and then provide a better traceability of the cryotubes, including timestamps that can validate the respect of the cold chain. With regard to Step 4, aliquots are first stocked in temporary boxes classed by organs and/or pathology types. Once full, these boxes are inventoried by two employees. The position of every cryotube (and of the sample it contains) is copied from the box's information sheet to the IS. This process can take up to 40 man-minutes. It is important to note that even if the tubes are barcoded, employees prefer 
to transcribe information, notably because of the frost on cryotubes. With an RFID reader, the reading time can be reduced to 6 seconds, even though a basic RFID reader cannot define the position of the cryotubes in the box. Nevertheless, new prototypes (so-called X/Y antennas) allow determining the position of each cryotube and thus transferring the related information directly to the IS. Such a reader would automate the manual copy of information from the datasheet to the IS and avoid copy errors. This reader has been observed inventorying boxes in 30 seconds. X/Y antennas also give an opportunity for a new activity within the biobank, consisting in the re-use of emptied sockets. Indeed, this activity needs short and effective inventory processes. This new activity will be detailed in Subsection 2.2 .

The accuracy of the IS is especially important when a handover request is processed (Step 5). If an error in a cryotube location occurs, the employee has to look for the missing cryotube at ambient temperature, which will slowly deteriorate the cryotubes. At the end-user reception point (Step 6), it is important to associate every cryotube sent with its information datasheet. An error during this process may distort the pharmaceutical research or make the diagnosis wrong and lead to reverse medical treatments. At the present time, the double checking principle is used at different steps to reduce all these errors. This principle reduces significantly error probabilities [Bonnabry 2007]. Inserting an RFID tag to every cryotube could make the biological samples and their related information inseparable. In this study, we focus on this way to use tags. Among the diverse possibilities of introducing RFID in biobank processes, we thus consider that tags are associated with all the tubes, including the arriving samples (Step 1) and cryotubes (Step 4), when aliquots are stored. The information leafs can be replaced by on-screen information, shall it be on a Wireless RFID reader or a workstation monitor. Recall that our study only considers the HF-RFID technology, as explained in Subsection 1.3.

All these observations make the biobank case interesting as it reflects the possibilities RFID can bring in more general contexts. The next subsection presents an activity enabled, or at least highly improved, by contactless technologies.

\subsection{A new activity: re-warehousing}

Despite the fact that the purpose of storing cryotubes in biobanks is eventually to use them in the future, and thus that their sockets can be expected to be cleared one day, reusing these sockets is not necessarily anticipated in biobanks. Actually, this possibility was not considered in the two biobanks involved in this study. A first reason was that the requests of cryotubes finally remained relatively unfrequent in these biobanks. A second reason was the reluctance to handle cryotubes and therefore expose them to ambient temperature. The forecasted increases both in the number of cryotubes to store and in the number of sample requests impose a better management of the storage capacity. Reusing emptied sockets then might become a necessity.

By improving the reliability of the information system and by allowing quick recognition of samples, RFID gives the possibility of reassigning safely tubes in sockets when needed. In this section, we investigate this issue. First, we describe the constraints to consider. Then, we discuss procedures to follow for this process. 
The main constraint, StayFrozen, deals with the time limit that a sample can be left out of its fridge. This constraint is the main reason why biobanks do not reallocate the emptied sockets for the time being. Indeed, without an effective technology, the duration of the inventory process and, consequently, the risks of deteriorating samples and making mistakes, are high. We denote $T_{M A X}$ the maximum acceptable time of exposure to ambient temperature for a cryotube. When this time is spent, the temperature of the sample is critical and it has to be stored back at low temperature immediately. Note that several assumptions are made here. First, we consider that the time limit $T_{M A X}$ does not depend on the sample type. Secondly, we do not take into account the fact that a cryotube does not recover its temperature immediately when stored back. Indeed, it is clear that time limit $T_{M A X}$ is excessive if a sample that has just been stored back is taken out again. With this assumption, we consider that the chances that a cryotube will repeatedly be taken in and out are very limited in an optimized process. Note that while this assumption seems reasonable for simulation, it certainly has to be carefully reconsidered for the real process.

A second constraint described by the practitioners, consists in maintaining the aliquots of a sample in the same box side by side. This constraint follows from existing organizational processes that ensure keeping at least one aliquot for a patient diagnosis. When introducing auto-ID technologies, the information system is however supposed to be sufficiently accurate to detect this case. For a better optimization of the storage capacity, we relax this constraint.

The third constraint is very similar to the previous one. The inventory is managed so that boxes only contain samples of a same type. This policy allows samples to be found easily when they are in transitory boxes. It allows also manipulating less boxes when numerous samples of the same kind are requested. Contrary to the previous constraint, RFID does not necessarily remove this constraint. As a consequence, it will be considered in our model and be called BoxesPerKind.

The last constraint is related to the maximum level of nitrogen in the room. When tanks are opened for storing or unstoring boxes, nitrogen evaporates. When a given level of nitrogen in the storage room is reached, an alarm warns the staff to evacuate. This constraint could be converted into a maximum number of boxes that could be stored or unstored when reassigning cryotubes in sockets. This constraint seems however rather marginal as long as the complete (or at least a large part of the) bank is not re-optimized. Consequently, we do not include it in our model.

Considering the above constraints, we propose the following scenario to perform the re-warehousing activity. It sounds secure and acceptable for health professionals:

-All boxes from/in which cryotubes will be removed/inserted are first taken out of the fridges by an employee;

- The employee then performs the moves of cryotubes that empty the maximum number of boxes, considering the StayFrozen and BoxesPerKind constraints; every move comes with an inventory update;

-Once finished, non-empty boxes are stored in the fridges;

Note that this procedure should also limit the evaporation of nitrogen and prevent from manipulating repeatedly some boxes in and out (as assumed when modeling in a simplified manner the StayFrozen constraint).

ACM: Transactions on Modeling and Computer Simulation, Vol. ?, No. ?, ?? 20??. 
Besides deciding how to proceed when the decision is taken to optimize the storage area, one has to define how this activity is triggered. We propose to apply the two following rules:

(1) A certain amount of time is scheduled in the week for this activity, e.g., every Tuesday evening (trigger 1, or also Planned trigger);

(2) All the tanks are full and the system needs to empty some boxes in order to accept new cryotubes (trigger 2, or also Emergency trigger).

Other possibilities would have been for example to consider the average level of fragmentation of the boxes, to detect complementary pairs of boxes (in the sense that the two boxes contain a total number of tubes of same type that precisely corresponds to the size of one box), to schedule re-warehousing in idle time periods, etc.

The literature on similar activities is very limited and only concerns the storage of products in industrial warehouses. According to [Garfinkel 2005], [Sadiq 1993] proposes the only approach addressing the problem of periodically reassigning stock items to storage locations. The author develops a heuristic method called "Stock Location Assignment Algorithm", based on the so-called HYCLUS clustering algorithm. The scarcity of literature on this subject can be explained by the fact that products are generally not identified at the item level. Furthermore (and consequently) a location is assigned for a long period of time to a category of products, products are picked up with policies like First In First Out (FIFO). Actually, storage locations mainly change with the arrival of new types of products. Decisions are then taken manually, without any decision-support tool.

\section{SIMULATION APPROACH}

Biobanks can be described as particular inventory management systems, happening in a complex environment, involving important reliability and safety issues as well as human operations. Incident recovery and alarm possibilities, automated control processes, sample arrival variability are dynamic parts of the system we must be able to model and observe. The presence of repetitive human operations also leads to a particular interest in Performance Shaping Factors (PSFs) modeling.

[Kleijnen 2005] distinguishes four kinds of simulation approaches that could be used for supply chain management and optimization: spreadsheet simulation, system dynamics, discrete event dynamic systems (DEDS) and business (or management) games. In the face of the difficulties to be tackled, DEDS appears as the most appropriate candidate for our study. [Kleijnen 2005] suggests that DEDS permits a more acute modeling than System Dynamics. Its abilities for including individual events and incorporating uncertainties are certainly attractive features considering our case study. [Borshchev and Filippov 2004] also suggest that DEDS is appropriate for mesoscopic studies of a system, like supply chains, call centers or emergency departments, even though it is not the only possibility.

A complementary possibility for studying the introduction of RFID in biobanks would have been to use Multi-Agent Systems, as different individual profiles can lead to different reactions, perception of alerts and processing adaptations. The importance of the interactions between employees is however relatively limited in the system we study and the effort required to develop a Multi-Agent System would not necessarily have been significantly rewarded compared to the use of DEDS only. 


\subsection{Process modeling}

Every process is described with a set of three subprocesses:

- The most important subprocess is the 'Medical act' (manipulation, cutting-off, transformation of samples, handover preparations, etc.).

- The second subprocess concerns the 'Information assets'. It consists for example in writing the status (date and hour), the type and the ID of the sample, the aliquot number, etc. The use of auto-ID technology will modify these subprocesses impacting the processing time and error probabilities.

- The last process is called 'Control'. Errors are the most critical problems in healthrelated production systems. As a consequence, most of the processes include control, generally through double checking: additional employees are requested to supervise error-prone activities. This multiple checking is supposed avoiding $85 \%$ of the incidents [Bonnabry 2007]. With RFID technologies, control subprocesses completely change, as double checking can be removed. Human resource utilization can be reduced and percentage of errors decreased.

These subprocesses are interconnected through precedence and coordination rules. For example, the 'Inventory box' process, happening when a transitory box is full and about to be stored, contains an 'Information assets' subprocess that consists in sending the inventory leaf to be written in the IS. Before starting, this subprocess needs the inventory process to be finished for the specified box. The 'Control' subprocess consists in double checking, which must be simultaneous to the 'Medical act'.

\subsection{Scenarios}

The model developed is designed to simply and automatically modify parameters when evaluating multiple scenario configurations. The following material scenarios are tested:

_.Current scenario" (Curr.Scenar), where paper sheets are associated with samples. No RFID technology is used. This scenario corresponds to the existing situation at the IPC and at the CHU-N.

- 'RNA Automaton" (RNA Auto.), where we added an RNA extraction machine. Without this machine, RNA extraction requires 3 hours of manual labor, outside the clean area of the biobank. The machine, that can be installed in the clean area, takes the same time but requires human actions only when launching the process, using 20 minutes of human time for 6 to 12 sample transformations. While not related to RFID, this scenario is implemented as a part of the validation of the simulation modeling. Indeed, the IPC has recently introduced these machines and is evaluating their impact.

—"Smart cryotubes and pen-size antenna"(RFID \& penRder), where RFID tags are attached to the tubes. Data sheet information are then accessible from the RFID labels, and the information are transmitted to the IS without manual copy. The pen-size antenna allows the inventory of boxes by pointing the cryotubes one by one. We estimated that the average time needed is reduced from 24 to 8 minutes, with one human resource instead of two.

_"Matrix reader's antenna" (RFID \& 2DRdr), which consists of using the $\mathrm{X} / \mathrm{Y}$ antenna instead of the pen-size antenna. Inventory of a whole box is then allowed in 2 minutes,

ACM: Transactions on Modeling and Computer Simulation, Vol. ?, No. ?, ?? 20??. 
including the time to set the box parameters in the appropriate software and inventory the box.

\subsection{Re-warehousing activity}

In this subsection we describe the re-warehousing activity. This activity being new, we have to entirely define its process. As explained in Section 2, it consists in three steps: taking out the selected boxes, moving tubes between boxes in order to empty boxes while updating the database information, and stocking the non-empty boxes in their tanks. This process involves three kinds of decisions for which we are going to define rules. The first type is to select the boxes to be taken out and their ordering. The second type is to define the set of tube movements. The last type is the ordering of non-empty boxes when stocking them back.

Let us first consider the box selection and ordering decisions. We consider that a given constant time $t_{b o x}$ is needed to destock or restock a box.

PROPERTY 3.1. An optimal solution exists where boxes that will receive tubes are destocked before boxes that will be emptied.

PROOF. First note that there is no interest in together adding and removing tubes from a box. Note also that removing tubes from a box is only interesting if it permits to empy the box. Boxes that are destocked thus either are emptied or receive new tubes (keeping the tubes initially present). For the sake of clarity, we introduce some notation. The destocking and restocking times of a box $b$ in a solution are denoted $t^{d}$ and $t^{r}$, respectively. A pair $\left(t, t^{\prime}\right)$ is said to be a pair of compatible dates if destocking a box at date $t$ and restocking it at date $t^{\prime}$ is valid against the StayFrozen constraint (considering the tubes initially present in this box). We draw the reader's attention on the fact that if a box $b_{i}$ receives tubes from a box $b_{j}$, then $\left(t_{j}^{d}, t_{i}^{r}\right)$ must be a pair of compatible dates.Consider an optimal solution and suppose that there exists two boxes $b_{i}$ and $b_{j}$, such that $b_{i}$ is emptied, and $b_{j}$ receives tubes. Suppose also that $b_{i}$ is destocked just before $b_{j}\left(t_{i}^{d}=t_{j}^{d}-t_{b o x}\right)$. In what follows, we will show that $b_{j}$ can be destocked before $b_{i}$ without deteriorating the initial solution. Four cases have to be considered,

(1) if tubes from box $b_{i}$ are moved into box $b_{j}$ then switching boxes $b_{i}$ and $b_{j}$ does not invalidate the initial solution ( $t_{j}^{r}$ does not have to be modified).

(2) if tubes from box $b_{i}$ are moved into a box $b_{k}$ that is destocked after $b_{j}$, then switching $b_{i}$ and $b_{k}$ does not invalidate the initial solution.

If all tubes of $b_{i}$ are moved into boxes that are destocked before $b_{i}$, then two situations have to be considered:

(3) if the time between destocking and restocking box $b_{j}$ is lower than or equal to $T_{M A X}-$ $t_{b o x}$ then box $b_{i}$ and box $b_{j}$ can be switched without invalidating the initial solution.

(4) if the time between destocking and restocking box $b_{j}$ is equal to $T_{M A X}$, then there exists a box $b_{k}$ such that $t_{k}^{d}>t_{j}^{d}$, which is restocked just before $b_{j}$. Then switching the destocking order of boxes $b_{i}$ and $b_{j}$ is possible, as soon as $t_{j}^{r}$ and $t_{k}^{r}$ can be inverted, and does not invalidate the solution.

By repeating this procedure we conclude that starting with boxes that receive tubes does not prevent from obtaining an optimal solution. 
PROPERTY 3.2. There exists an optimal solution where the destocking/stocking orderings follow a FOFI (First Out First In) rule.

PROOF. Consider an optimal solution with the boxes to be emptied closing the destocking sequence. Suppose there exists two boxes $b_{i}$ and $b_{j}$ such that $b_{i}$ is destocked before $b_{j}\left(t_{i}^{d}<t_{j}^{d}\right)$ and is restocked after $\left(t_{j}^{r}<t_{i}^{r}\right)$. Dates $t_{i}^{d}$ and $t_{i}^{r}$ are compatible and thus are dates $\left(t_{i}^{d}, t_{j}^{r}\right)$ and dates $\left(t_{j}^{d}, t_{i}^{r}\right)$. Also for every emptied box $b_{k}$ (from which tubes might have been added to $b_{i}$ or $\left.b_{j}\right), t_{k}^{d}>t_{j}^{d}$, which implies that $\left(t_{k}^{d}, t_{j}^{r}\right)$ and $\left(t_{k}^{d}, t_{i}^{r}\right)$ also form pairs of compatible dates. From these inequalities, one can see that $b_{i}$ and $b_{j}$ can be inverted in the restocking sequence. Property 3.2 follows by repeating this mechanism until the restocking sequence repeats the destocking ordering.

So as to define sensible rules for the tube movements and to compute an upper bound, we analyze the simplified case where the time to manipulate the boxes is neglected. We define $t_{t u b}$ the time needed to move a tube between two boxes (including the inventory update), which is also assumed to be constant. With these assumptions, the StayFrozen constraint can be expressed as a limit on the number of tube movements. The problem to consider is then to find a set of moves such that a maximal number of boxes is emptied, with constraints limiting the number of movements (StayFrozen constraint) and implying that moves only concern boxes of the same type.

We propose to apply the following algorithm. At every iteration of the algorithm, a box is emptied, until the limit on the number of moves and/or the loading rate of the boxes prevent from emptying any additional box. The box selected to be emptied is the one with the minimal number of tubes among the boxes that can be emptied. A box can be emptied when there exist boxes of the same type that are able to receive the tubes. The tubes are then moved to these boxes, in the decreasing order of their loading rate.

PROPERTY 3.3. Under the assumptions we made in the preceding paragraph, the above algorithm is optimal.

PROOF. Let consider an optimal solution and note $B_{-}$the set of boxes that have been emptied and $B_{+}$the set of boxes where tubes have been added. Note $q_{i}$ the initial number of tubes in a box. Let us assume that there exists a box $b_{i} \in B_{-}$and a box $b_{j} \in B_{+}$of the same type with $q_{j}<q_{i}$. Another optimal solution can be constructed by removing $q_{j}$ tubes from box $b_{j}$ and $q_{i}-q_{j}$ from box $b_{i}$, instead of $q_{i}$ tubes from box $b_{i}$, and by adding tubes to box $b_{i}$ instead of box $b_{j}$. Box $b_{j}$ will then be emptied and box $b_{i}$ will be able to receive the new tubes as its load once the tubes are removed becomes $q_{j}$. Note that the solution should still be modified to avoid adding and removing tubes from box $b_{i}$, which can be simply done by removing the intermediary transit to box $b_{i}$.

Repeating this mechanism, we obtain a solution where tubes are removed from boxes with minimal loading rates only. The solution can then easily be transformed so that the tubes are only moved to the boxes with the maximal loading rate. Note that this last part of the policy does not minimize the number of boxes involved in the process: choosing the non emptied boxes with minimal loading rates would allow less boxes to be involved. However, it might help to empty new boxes later on.

Let us now return to the case where box manipulation times are not neglected. Since it solves a relaxation, the above algorithm provides an upper bound on the number of boxes 
that can be emptied in one execution of the activity. To find a feasible solution that maximizes the efficiency of the process, we proceed as follows. We empty boxes as detailed in the algorithm but the boxes selected for receiving the tubes are the following ones in the order of increasing loading rate. This policy minimizes the number of boxes to be destocked and optimizes the chances that the solution is feasible regarding the StayFrozen constraint. The feasibility of the solution is then evaluated. If feasible, the solution is returned. Otherwise, a solution with one less emptied box is investigated. The tube movements that empty the last box to be emptied are canceled, and this box become a target box. The algorithm is repeated until a feasible solution is found. Being rather intuitive and copying what practitioners might tend to execute, this algorithm is subsequently called "Intuitive" algorithm. This algorithm is carried out every time re-warehousing is planned (trigger 1). When rewarehousing is done in emergency (trigger 2), the process is different. We only consider the subset of tube movements that empty the first box. We then construct the corresponding solution and evaluate its feasibility. If feasible, we empty the box and stop. Otherwise, an emergency rack starts being used and a new rack is ordered. In this case, a certain execution time $T_{\text {shortage }}$ is defined for the administrative work.

Though being the most efficient for one occurrence of re-warehousing, the Intuitive algorithm might not have the best results in the long run, as leveraged in the proof of Property 3.3. Moving tubes to the non-full boxes having a maximal number of tubes as proposed could however be a little bit too extreme as the number of boxes involved might become detrimental. We rather propose the following scheme, coined as the "Long Term" algorithm. First, the set $B_{1}$ of boxes that would be emptied relaxing the Stayfrozen constraint is computed. Boxes of $B_{1}$ then represent boxes from which tubes could be removed, while the complementary set of boxes, $B_{2}$, represent boxes where tubes could be added. The algorithm then manages to empty a box of $B_{1}$ with a maximal number of tubes to boxes of $B_{2}$ with maximal numbers of tubes. Note that a single box is emptied with this algorithm. Intuitively, this algorithm is designed so that opportunities for emptying boxes in the next emergency re-warehousings are maximized.

\subsection{Performance indicators}

Evaluating the performance of a health service is a complex issue [Minvielle et al. 2007]. The main points to consider are:

- The safety of patients and employees;

-Patient satisfaction;

-Financial impact;

—Employee reluctance and/or understanding.

For our study, we considered the set of following indicators:

Number of errors. This indicator is split in two parts: number of computation errors, number of inventory errors.

Fastness of processing times. Three indicators are defined with regard to processing times. A first indicator, IS Fulfillment delay, provides the amount of time spent between the reception of the sample and the computerization of its related information. In our study, improving this indicator might accelerate the time of obtaining analysis results, which is 
important for the safety of the patients. Transformation time is the second indicator, giving the time between the reception of the samples and the computerization of their transformed aliquots. The third indicator, Throughput time, provides the time between the reception of the samples and the time when their transformed aliquots are put in the fridge.

Human utilization. This indicator gives the number of man-minutes required by the processes. It does not only reflect a financial impact, but also working conditions for the employees. Biobank activities are indeed not really satisfying for employees, as they do not call for their specific abilities ([Swedberg 2009], for example, describes a case where the motivation of trained employees increases when machines are used to automate repetitive tasks that do not require high qualifications).

Storage racks bought. This indicator gives the number of storage racks that must be bought to follow the evolution of the biobank activity. This indicator could be translated to a financial indicator, by taking different parameters into account, such as rack costs, storage costs, storage room capacity, tank capacity, etc.

Note that no indicator is specifically dedicated to the financial impacts, as they can be very dependent on the structure. Health structures indeed may have different policies regarding the development of their storage area; some of the devices we are considering are prototypes that may force biobanks to invest in whole proprietary solutions; the workforce costs are highly variable depending on the region of the world; the costs of emerging technologies are not stabilized yet...

\section{EXPERIMENTATIONS}

\subsection{Implementation}

To implement our model, we use the Arena software, described by [Law and Kelton 2000] as a general purpose simulation software, allowing the creation of customized modules, templates, and user friendly front ends. It can be easily connected with other softwares through ActiveX [Seppanen 2000], and encodes user functions through VBA blocks. The use of Dynamic Link Library (DLL) is useful to plug-in external algorithms that may be reused elsewhere, like in real health structures for example.

The employees are modeled using simple resources, which can be 'Available', 'Busy' or 'Unreachable'. VBA blocks are user defined blocks used to fill the databases and assign random generated attributes like errors, process durations, number of aliquots in samples, arrival or demand of samples, etc. We use these blocks to improve the way the resources are modeled and for inventory management. We also implement a basic PSF based on the number of samples waiting to be carried out. We consider that a large amount of pending jobs increases the error rate by $5 \%$.

Most of the specific functions are implemented in VBA as recommended by [G.F.Kalsdorf 2006]. Our goal being to compare material and organizational configurations, we use a spreadsheet to define the scenarios, that is, device behaviors, process lengths and error probabilities. For example, introducing an RFID reader at the reception point will reduce processing times of some of its subprocesses and error probabilities. Other dysfunctions than human failures are implemented; device or network crashes are possible, even if the

ACM: Transactions on Modeling and Computer Simulation, Vol. ?, No. ?, ?? 20?? 
Table I: Time to walk from an area to another (in seconds).

\begin{tabular}{|l|c|c|c|c|}
\hline To From & Reception area & Transformations area & Storage area & NRA/DNA extraction area \\
\hline 1- Reception area & 0 & 21 & 19 & 123 \\
\hline 2- Transformations area & 21 & 0 & 33 & 103 \\
\hline 3- Storage area & 19 & 33 & 0 & 144 \\
\hline 4- NRA/DNA extraction area & 260 & 260 & 260 & 0 \\
\hline
\end{tabular}

probability is very low. In case of crash, employees are then obliged to access manually to the databases and to re-label the tubes. As it contains both material and organizational parameters, the spreadsheet allows fast scenario configurations.

\subsection{Data}

In this subsection, we describe the data used for the simulations. These data were given or validated by health professionals, or observed through demonstrators set up during the MISTRALS project.

The time between arrivals of new samples is exponentially distributed. The opening hours include peak hours, i.e. a time interval, during which the arrival rate is larger than during the rest of the day. These values are computed from the total number of yearly incoming samples divided by the time the structures are opened. The arrival is increased by a ratio of 1.618 during the peak hours, which is an arbitrary value considered realistic by health professionals. The handover request arrivals are exponentially distributed, with a mean of 1 day. In case of arrival, the number of tubes depends on a uniform law of mean 5.5 , limited to the interval $[3,8]$. For each tube, the handover generator first selects a box, then a used socket in this box.

A box of cryotubes contains 100 sockets. Boxes are stocked in racks, each rack can contain up to 12 boxes. The time needed to use a new rack, $T_{\text {shortage }}$, is set to half an hour. For every replication of the simulation, all the scenarios start with an identical initial stock. The three types of stock sets which were used are described thereafter.

A first type of initial stock ("EmptyStock") contains only two full racks of full boxes, so the first box to be stored will probably lead to using a new rack. For the two other cases, boxes are generated until the stock contains at least 100,000 tubes. The number of tubes in each box is decided using a uniform probability function. For the first case, which leads to a $77.5 \%$ filling level mean, each box has a $10 \%$ probability to contain between 11 and 60 tubes, $60 \%$ to contain 61 to 90 tubes and $30 \%$ to contain 91 to 100 tubes. The probabilities in these ranges are uniform. The second initial stock level mean is $81 \%$, for which the probabilities are the same but the ranges are: 11 to 70 tubes, 71 to 90 tubes and 91 to 100 tubes. These stock levels were determined for flanking the limit when it becomes impossible not to buy any rack, while the bounds are estimated to create realistic initial inventories.

The transformation processing times have been implemented using data from the IPC. Table I indicates the times needed to move between rooms; the matrix is not symmetric because there are differences between the operations required when entering and leaving the clean area. Re-warehousing is planned every Friday afternoon. Re-warehousing parameters are defined as follows (in seconds): $T_{M A X}=1800, t_{b o x}=150, t_{t u b}$ depends on the scenario, $t_{t u b}=3$ when using $\mathrm{X} / \mathrm{Y}$ antenna and $t_{t u b}=5$ when using a pen-size antenna.

The basic probability of making errors when transcribing a data file by hand on a computer is set to $1.1 \%$. This value comes from the multiplication of the two following values: 
[Marcellis-Warin 2003] reports that 10\% of the operations in hospitals are subject to errors and that $11 \%$ of these errors are due to retranscriptions. This probability is reduced to $0.01 \%$ when tubes are tagged as data are automatically transferred.

The probability that an error occurs while manually inventorying a full box of cryotubes is set to $3 \%$. This value was estimated after discussing the results of [Garnerin et al. 2007] with health professionals. This value is set to $0.01 \%$ when the employees use an RFID pen-size antenna to perform the inventory.

These probabilities are divided by the employee Performance Level. The performance level is identical for every employee during a replication of the simulation; the values are either $0.5,1$, or 2 . Comparing different values of this cursor allows us to observe the relative impact of RFIDs depending on the qualification level of employees, the lack of training that can happen in some institutes / countries, or the use of third-party resources. It would be interesting, for future research, to vary this level throughout the day and/or from one day to another, depending on factors such as fatigue or psychological profile modifications.

Table II gives the values of the scenario main parameters (scenarios are described page $10)$ :

—times of the "Information asset" subprocess (I ProcessName).

- times of the "Control asset" subprocess (C_ProcessName);

—error probabilities (Perror_TaskName);

—number of resources required for certain processes (HumRess_TaskName);

—characteristics of DNA/RNA operations;

Table II: Scenario main parameters.

\begin{tabular}{|l|r|r|r|r|}
\hline Scenario / Parameters & Curr.Scenar & RNA Auto. & RFID \& penRdr & RFID \& 2DRdr \\
\hline IReceptTime (min.) & 0.75 & 0.75 & 0.25 & 0.25 \\
\hline ITransformation (min.) & 1 & 1 & 0.5 & 0.5 \\
\hline CInventory (min.) & 20 & 20 & 0 & 0 \\
\hline HumRessCInventory & 1 & 1 & 0 & 0 \\
\hline PerrorSampleLeaf & $1.1 \%$ & $1.1 \%$ & $0.001 \%$ & $0.001 \%$ \\
\hline PerrorInventory & $3 \%$ & $3 \%$ & $0.1 \%$ & $0.01 \%$ \\
\hline HumRessComputerizeLeaf & 1 & 1 & 0 & 0 \\
\hline HumRessTransportLeaf & 1 & 1 & 0 & 0 \\
\hline AreaRNAProcess & 4 & 2 & 2 & 2 \\
\hline SetupRNA (min.) & 170 & 20 & 20 & 20 \\
\hline WaitRNAProcess (min.) & 0 & 60 & 60 & 60 \\
\hline FinishRNA (min.) & 0 & 20 & 20 & 20 \\
\hline$t_{t u b}$ (sec.) & N/A & N/A & 5 & 3 \\
\hline
\end{tabular}

Unless the contrary is explicitly stated, the experiments are conducted with an employee Performance Level of 1 and the "Empty stocks" scenario for initial stock levels. The time horizon defined for the simulations is 52 working weeks.

ACM: Transactions on Modeling and Computer Simulation, Vol. ?, No. ?, ?? 20?? 


\subsection{Results}

The number of replications was determined following the recommendation dispensed by [Kelton et al. 2007]. The half-width of the resource Human utilization observed after 25 replications led to a recommendation of 48 replications to be sure at $95 \%$ that this value would vary for less than $0.2 \%$. We decided to run 50 replications. Results are given in tables III, IV and V.

The notation corresponding to the scenarios in the result tables is as follows: Curr. Scenar is the case with no RFID nor RNA automaton. In RNA Automaton, a DNA/RNA automaton was added, which is also present in all scenario including RFID. Pen-Rder and $2 D R d e r$ correspond respectively to the use of a pen-size antenna or an $\mathrm{X} / \mathrm{Y}$ antenna during the inventory process. The non-RFID scenarios do not include the re-warehousing activity. The Re-Wa argument corresponds to the case when the emergency trigger is enabled as well as a weekly planned trigger. In this case, boxes must contain only tubes of the same kind; the "No BPK" argument explicits that this constraint is removed. The Emergency only argument corresponds to the case when there is no scheduled re-warehousing. The algorithm used for re-warehousing is the Intuitive Algorithm described in Section 3.3, unless lengTerm is assed and the long term algorithm is used.

Table III focuses on the results regarding the main configurations. It compares the nonrewarehousing scenarios: "Curr.Scenar", "RNA Auto.", "RFID \& penRder \& No re-Wa", "2DRder \& No re-Wa". It also shows the results of the referent re-warehousing scenario: "2DRder \& Re-Wa" and its variation while using a pen-reader antenna: "penRder \& ReWa". These scenarios are compared through the Fastness of processing times, Number of errors, Human utilization and Storage racks bought.

Table III: Performance indicators for main configurations.

\begin{tabular}{|c|c|c|c|c|c|c|c|}
\hline $\begin{array}{c}\text { Scenario } \\
\text { description }\end{array}$ & \multicolumn{3}{|c|}{ Processing Times } & \multicolumn{2}{c|}{ Errors } & \# Racks & Hum. Ress. \\
\hline Is Fulfillment & Transformation & Throughput & Computerization & Inventory & Bought & Used \\
\hline Curr.Scenar & $3.06 \mathrm{~h}$ & $5.94 \mathrm{~h}$ & $2.09 \mathrm{~h}$ & $1.1 \%$ & $3.03 \%$ & 24.09 & $51.74 \%$ \\
\hline RNA Automaton & $2.69 \mathrm{~h}$ & $5.27 \mathrm{~h}$ & $1.71 \mathrm{~h}$ & $1.15 \%$ & $2.96 \%$ & 24.24 & $45.21 \%$ \\
\hline Pen-Rder \& No re-Wa & $0.35 \mathrm{~h}$ & $1.63 \mathrm{~h}$ & $1.61 \mathrm{~h}$ & $0.01 \%$ & $0.15 \%$ & 24.19 & $32.37 \%$ \\
\hline 2DRder \& No re-Wa & $0.35 \mathrm{~h}$ & $1.62 \mathrm{~h}$ & $1.61 \mathrm{~h}$ & $0.01 \%$ & $0.01 \%$ & 24.12 & $31.61 \%$ \\
\hline pen-Rder \& Re-Wa & $0.35 \mathrm{~h}$ & $1.63 \mathrm{~h}$ & $1.61 \mathrm{~h}$ & $0.01 \%$ & $0.06 \%$ & 19.86 & $32.61 \%$ \\
\hline 2DRder \& Re-Wa & $0.35 \mathrm{~h}$ & $1.62 \mathrm{~h}$ & $1.61 \mathrm{~h}$ & $0.01 \%$ & $0.01 \%$ & 19.84 & $31.93 \%$ \\
\hline
\end{tabular}

The percentage of Human Resource Utilization in the initial scenario Curr.Scenar is $51.74 \%$, which can be explained considering that employees spend a large amount of time working for other services and that the amount of work depends on the arrival rate, which is generally irregular. Slightly more than $10 \%$ of this time is saved when adding the RNA automaton in the clean area (Human Resource Utilization of 45.21\%). The introduction of RFID has still more impact on this indicator with an additional decrease of almost $31 \%$. Introducing the re-warehousing activity has the opposite effect; the additionnal time spent for this activity appears however very limited (less than $0.5 \%$ ) and is largely conterbalanced with the savings in rack utilizations (decrease of $20 \%$ ). Table $\mathrm{V}$ will analyze further this latter indicator showing that even more significant results can be obtained with different initial stock levels.

With regard to Processing Times, similar results can be observed. IS Fulfillment delays are 
specially reduced with RFID, as initially consisting mainly in handwriting, data keyboarding and information leaf transportation that can almost entirely be removed. Note that this reduction is all the more interesting that these tasks are far from the core competences of the employees. Throughput times reveal that the savings in time also result in accelerated handling operations for incoming samples, which is also an important indicator for quality matters. A final worth-mentioning point about processing times is the small influence of the type of RFID reader (pen or X/Y antenna) that does not make significant differences.

Table IV analyses the effect of the employee Performance Level (EPL) on inventory and computerization errors. Scenarios involving re-warehousing are not included here as error rates are not significantly affected by this activity.

Table IV: Observed errors on the non-rewarehousing scenarios, depending on Employee Performance Level (EPL).

\begin{tabular}{|l|c|r|r|r|r|r|r|}
\hline \multicolumn{2}{|c|}{ Scenario } & \multicolumn{3}{|c|}{ Inventory error } & \multicolumn{3}{c|}{ Computerization error } \\
\hline Material configuration & $E P L$ & Avg. & Min. & Max. & Avg. & Min. & Max. \\
\hline Curr.Scenar & 0.5 & $6.02 \%$ & $3.45 \%$ & $9.54 \%$ & $2.23 \%$ & $1.81 \%$ & $2.65 \%$ \\
\hline Curr.Scenar & 1 & $3.03 \%$ & $0.72 \%$ & $4.98 \%$ & $1.1 \%$ & $0.81 \%$ & $1.39 \%$ \\
\hline Curr.Scenar & 2 & $1.51 \%$ & $0.35 \%$ & $3.46 \%$ & $0.57 \%$ & $0.37 \%$ & $0.8 \%$ \\
\hline RNA Automaton & 0.5 & $6.27 \%$ & $2.91 \%$ & $10.14 \%$ & $2.3 \%$ & $1.96 \%$ & $2.64 \%$ \\
\hline RNA Automaton & 1 & $2.96 \%$ & $1.06 \%$ & $5.28 \%$ & $1.16 \%$ & $0.94 \%$ & $1.49 \%$ \\
\hline RNA Automaton & 2 & $1.5 \%$ & $0 \%$ & $3.16 \%$ & $0.57 \%$ & $0.41 \%$ & $0.79 \%$ \\
\hline RFID \& pen-Rder & 0.5 & $0.25 \%$ & $0 \%$ & $1.03 \%$ & $0.02 \%$ & $0 \%$ & $0.06 \%$ \\
\hline RFID \& pen-Rder & 1 & $0.15 \%$ & $0 \%$ & $1.05 \%$ & $0.01 \%$ & $0 \%$ & $0.05 \%$ \\
\hline RFID \& pen-Rder & 2 & $0.1 \%$ & $0 \%$ & $0.7 \%$ & $\leq 0.01 \%$ & $0 \%$ & $0.03 \%$ \\
\hline RFID \& 2DRder & 0.5 & $0.1 \%$ & $0 \%$ & $0.36 \%$ & $0.02 \%$ & $0 \%$ & $0.08 \%$ \\
\hline RFID \& 2DRder & 1 & $0.01 \%$ & $0 \%$ & $0.35 \%$ & $0.01 \%$ & $0 \%$ & $0.05 \%$ \\
\hline RFID \& 2DRder & 2 & $\leq 0.01 \%$ & $0 \%$ & $0.35 \%$ & $\leq 0.01 \%$ & $0 \%$ & $0.06 \%$ \\
\hline
\end{tabular}

The table shows the high impact of the employees Performance Level when RFID is not present (Curr.Scenar, RNA Automaton). Improvements are less significant when autoID technologies are introduced as many critical processes like handwritings or manual computerizations are removed. This result is important as it shows that RFID technologies are more profitable to organizations where the staff is less qualified (or overloaded), that is, most likely, the ones that cannot afford these technologies.

Table $\mathrm{V}$ focuses on the impacts of re-warehousing decisions. The scenarios evaluated in this table all derive from a single scenario, the so-called referent scenario, namely $2 D R d e r \& R e-W a$. Every scenario is obtained by modifying exactly one aspect of the referent scenario: change the XY antenna to a pen-reader, relax the BoxesPerKind constraint (thus allowing different types of tubes in a same box), disable the weekly planned re-warehousings, use the Long Term algorithm while applying re-warehousing. In this last case, the effect of changing from a weekly planned re-warehousing to a daily planned rewarehousing is also investigated. The results for all these scenarios are reported for the three initial stock levels detailed in Section 4.2. They include:

- The average number of emergency triggers launched (Number Launched) and the average number of times when it did not permitted to empty a box (\# Failed), as well as the 
standard deviation for this indicator. The average value for \# Failed is written in bold as it exactly indicates the number of racks that had to be bought.

- The average number of boxes emptied during planned re-warehousings (\# Free Boxes) and the number of scheduled re-warehousing that were canceled since no box could be emptied; in this case it is important to understand that the processing time for the re-warehousing was then saved. 


\begin{tabular}{|c|c|c|c|c|c|c|c|}
\hline & 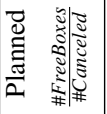 & 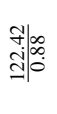 & 泀| & 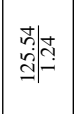 & $\mathbb{z}$ & 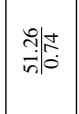 & م| \\
\hline$\frac{0}{\infty}$ & 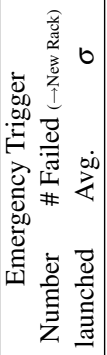 & 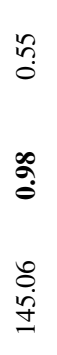 & 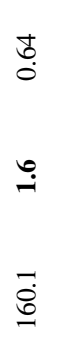 & 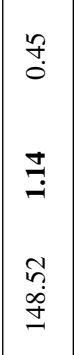 & 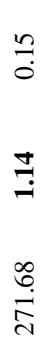 & 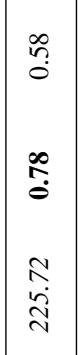 & 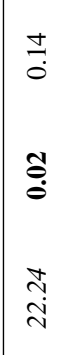 \\
\hline & 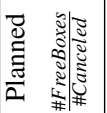 & î:0 & $\begin{array}{l}\bar{b} \\
\dot{a} \\
\mid\end{array} \mid 0$ & 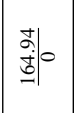 & $\mathbb{z}$ & inlo & भîे| \\
\hline 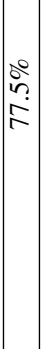 & 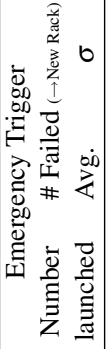 & $\frac{\dot{0}}{0}$ & $\begin{array}{l}0 \\
\circ \\
\infty \\
\stackrel{1}{~}\end{array}$ & $\frac{ \pm}{8}$ & 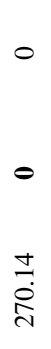 & $\begin{array}{l}0 \\
\vec{b} \\
\infty \\
\vec{N}\end{array}$ & $\begin{array}{l}0 \\
2 \\
\dot{0} \\
\dot{2}\end{array}$ \\
\hline & 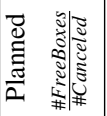 & 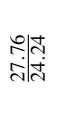 & 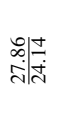 & 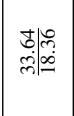 & $\mathbb{z}$ & 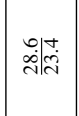 & 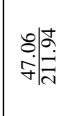 \\
\hline 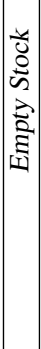 & 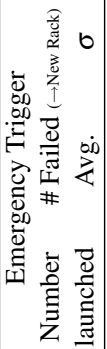 & 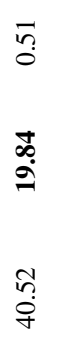 & 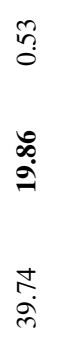 & $\begin{array}{l}\stackrel{9}{0} \\
\text { 워 }\end{array}$ & 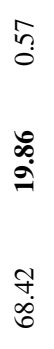 & 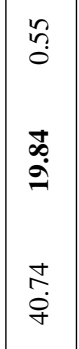 & 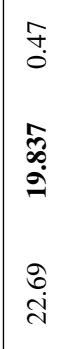 \\
\hline 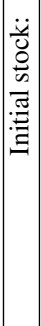 & 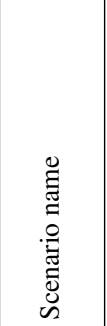 & 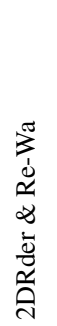 & 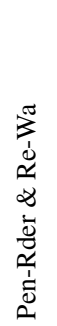 & 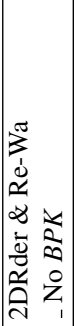 & 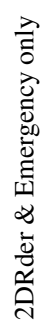 & 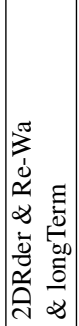 & 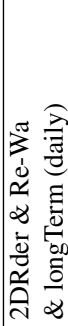 \\
\hline
\end{tabular}

ACM: Transactions on Modeling and Computer Simulation, Vol. ?, No. ?, ?? 20??. 
A first tendency drawn by Table $\mathrm{V}$ concerns the impact of the initial stock levels. Whatever the scenario, about 20 new racks are needed for the Empty stock case, while no racks need to be bought for the "77.5\%" initial level and a very limited number of racks (varying around 1) are bought in the " $81 \%$ " case. "Empty stock" corresponds to the worst situation for re-warehousing, where every box is full at the beginning of the simulation (or samples in boxes are not tagged yet). With an initial level of "77.5\%" on the contrary, empty places in the boxes are sufficiently numerous so that buying new racks is never needed. An initial stock level of "81\%" defines a compromise between these two extreme situations. Note that these values of course depend on the sample arrival and handover request rates that were defined for the simulation. An important phenomenon is however not explicitly shown in the table: the initial stock level scarcely impacts the number of new tracks needed when no re-warehousing policy is implemented. Indeed, empty places present in the storage area can no longer be exploited in this latter case (which actually corresponds to the actual situation). Whatever the initial stock level, the number of new racks needed would approximately attain 24 , as shown by Table III. In the long term, once a significant number of tubes are tagged in the biobank and as long as sample arrivals and handover requests exist, Table $\mathrm{V}$ demonstrates that re-warehousing is unavoidable and successful for limiting the growth of the biobank without pruning sample sets.

The comparisons of the different scenarios against the referent scenario also show interesting results.

With regard to the readers (2DRder \& Re-Wa against Pen-Rder \& Re-Wa), by allowing inventorying boxes more quickly than a pen-reader, $\mathrm{X} / \mathrm{Y}$ antennas permit to empty more boxes while performing re-warehousing. As a consequence, emergency re-warehousings also happen less frequently. However, the effect on the number of needed racks remains rather limited.

Removing the BoxPerKind constraint (2DRder \& Re-Wa against $2 D R d e r \& R e-W a{ }_{-} N o$ BPK) has a surprising effect at first sight. While one would expect more effectiveness, results are at best slightly improved and sometimes deteriorated. The reason for this counterintuitive result stands in the fact that a single transitory box is used in this case (compared to a transitory box per type otherwise).

When re-warehousing is only triggered in emergency (2DRder \& Re-Wa against $2 D R d e r$ \& emergency only), the number of emergencies largely increases, as expected. In the worst case for example ("77.5\%" intitial stock level), emergencies pass approximately from 2 per week to 5 per week. At the same time, this trigger appears to be sufficiently effective to limit the number of new tracks needed. Intuitively, one can understand that the use of the storage area can be optimized rather equivalently with the two triggers, but the moments in times where the optimization happens are different: a relatively long time every week with the planned trigger, numerous unpredictable short times with the emergency trigger.

The last scenario investigated concerns a modification of the algorithm used for re-warehousing (2DRder \& Re-Wa against 2DRder \& Re-Wa \& long Term). As explained in Section 3.3, the Intuitive algorithm has a myopic view on the optimization: many boxes might tend to be half empty while a collection of almost empty and almost full boxes would be preferable. The Long Term algorithm is designed to amend this behavior. With this algorithm, less boxes are emptied ( 1 box per planned re-warehousing instead of more than 2 or 3, on average, with the Intuitive algorithm for the " $81 \%$ " and "77.5\%" stock level scenarios, respectively), the number of emergencies increases, but the re-assignment of tubes is more 
effective and less additional racks are needed. A daily planning of the re-warehousing activity still highlights the impact of the Long Term algorithm, keeping nearly always 1 emptied box per planned re-warehousing but also allowing removing most of the emergencies and almost avoiding purchasing any rack.

\subsection{Discussion}

Our results do not include a formal financial part. The following indicators: Human utilization variation, Number of racks bought, number of smart-tubes used, costs of new device maintenance, are the most significant variable costs, while the readers, antennas and their installation costs, IS update, hardware, software and consulting are fixed costs. The staff training requires an initial investment, but their efficiency using new technologies will anyway evolve over time, as for example the "double labeling" will not be given up at first. Staff members will require an initial training for these technologies but this is already the case with barcodes, although the manipulation of mobile readers may require a short time of adaptation and the processing times may evolve with their experience with new technologies (tactile interface, graphical interface, etc.).

The use of RFID technologies leads to significantly reduce the number of racks that had to be used when considering that the whole stock was previously tagged. The "EmptyStock" scenarios show that, according to the handover arrival implemented, an important proportion of racks can be saved in the first year of use. The handover requests should, to validate this results, evolve over time, and depend on the stock content. This parameter also depends on the policy biobank managers want to apply and on the easiness to convince patients to give their authorization for using their samples.

It is important to remind that some observations of the studied system are needed to validate our assumptions. This part of our work will only be possible after the pilot plant have been observed long enough to become stabilize. On a technical part, the probabilities of sample misreading, chip failure and for the tube to lose its chip will have to be observed in their real condition of use to validate the assumptions. Concerning the estimated times for re-warehousing, we will be able to precisely observe the times for manipulating tubes and inventorying boxes. The boxes manipulation time (storing or unstoring) could also be better defined but it would require one additional manipulation or device integration (for tracking the employees position, for example). Other parameters may impact the data used in this study, like the temperature in the storage area impacting $T_{M A X}$. We also point out the fact that some solutions for tagging cryotubes may not be applicable to existing banks.

The assumptions concerning errors probabilities cannot be observed afterwards and will stay theoretical as the comparisons between database information and the physical stock would require a large number of observation to be relevant. The values of device failure and manipulation times will be observed when the pilot plant is operational. An interesting observation will be the employee adaptation time where we may observe a first transition when employees use the devices more easily. Then a second phase could come, during which urgent needs and daily emergencies may jeopardize the robustness of the system.

\section{CONCLUSION}

In this paper, we estimated the impact of RFID technologies in a particular healthcare application using discrete-event simulation as a decision support tool. Our study quantifies the benefits of integrating new auto-ID technologies and devices, addressing the possibility

ACM: Transactions on Modeling and Computer Simulation, Vol. ?, No. ?, ?? 20?? 
to enable new activities. We proposed a modeling approach which could be transposed in other (health) production systems.

The results show that, according to the parameters, auto-ID technologies may considerably improve the performances of biobanks when taking into account the percentages of errors as well as the reduction of used resources and processing times as performance indicators.

The proposed model should allow us to model other biobanks and/or material configurations quite quickly, including organ sample arrivals. This can be made using the same basic model file and only modifying the specific configuration file. These types of tools could be very helpful when auditing and expertizing facilities that must adopt new technologies because of new legislations, or because their maximum capacity is reached for example.

This paper addresses the re-warehousing activity, which may become a necessity in some facilities. We compared some policies concerning a possibly new activity which aims at optimizing the stock. Though the algorithm and scheduling of this activity could be improved, our observations show its implementation can lead to high savings.

Improving this study could consist in taking into account more constraints and triggers for the re-warehousing processing, defining an optimal planning considering the handover requests probabilities, or more manipulation scenarios (several technicians collaborating). A graphical user interface could allow biobank managers to model themselves their biobank; and enable the possibility of investigating the impacts of a global adoption of this technology. Improving the way human resources are modeled, using a multi-agent system and adding Performance Shaping Factors and trying different psychological profiles for example, would also be very interesting.

\section{ACKNOWLEDGMENTS}

This study is partially financed by the Conseil Général 13 of the Bouches du Rhône. MISTRALS was labelled by the PAC-ID cluster and is funded by the DGE/MINEFI. The authors gratefully thank all the collaborators of the MISTRALS project who always answered quickly and carefully the questions, and particularly Lise-Marie Daufresne, from the IPC, and Olivier Bordonne, from the CHU-N. The authors also thank the reviewers for their acute observations and propositions that improved the quality of this paper.

\section{REFERENCES}

AndrechaK, G. And WiEns, R. A. 2006. Hitachi -chip rfid technology compatible with gamma sterilization. Available via http: / / www. mds. nordion.com/ [accessed April 6, 2009].

ARumugAM, D. AND ENGELS, D. 2009. Specific absorption rates in the human head and shoulder for passive uhf rfid systems. Int. J. Radio Frequency Identification Technology and Applications 2, 1, 1-26.

Bettendorf, E., Malenfant, C., And Chabannon, C. 2008. RFID technologies and electronic tags to identify cryopreserved materials.

Boginski, V., Mun, I., Y. WU, K. M., AND ZHANG, C. 2007. Simulation and analysis of hospital operations and resource utilization using RFID data. In IEEE International Conference on RFID, Grapevine, Texas, USA, pp 199-204.

BONNABRY, P. 2007. How can the labelling and the packaging of drugs impact on the drug safety? In Swissmedic, Bern, Suisse. 
Borshchev, A. AND Filippov, A. 2004. From system dynamics and discrete event to practical agent based modeling: Reasons, techniques, tools. In Proceedings of The 22nd International Conference of the System Dynamics Society, July 25-29, Oxford, England.

CAuchois, R. 2008. Développement de tests de vieillissment accéléré de puces rfid conservées pour application en biobanques. Tech. Rep. ENSM-SE CMP WP 2008/01, École des Mines de Saint-Étienne.

Dalton, G. D., Samaropoulos, X. F., And Dalton, A. C. 2008. Improvements in the safety of patient care can help end the medical malpractice crisis in the united states. 86, 153-162.

DE KoK, A., VAn DonselaAR, K., AND VAN Woensel, T. 2007. A break-even analysis of rfid technology for inventory sensitive to shrinkage. Int. Journal of Production Economics 112, 2, 521-531.

E CANCER.FR. 2007. http: //www. e-cancer. fr/ [accessed october 30, 2009].

GARFINKEL, M. 2005. Minimizing multi-zone orders in the correlated storage assignment problem. Ph.D. thesis, Georgia Institute of Technology.

Garnerin, P., Pellet-Meier, B., Chopard, P., Pergener, T., and Bonnabry, P. 2007. Measuring human-error probabilities in drug preparation : a pilot study. Eur J. Clin Pharmacol 63, 769-776.

Gaukler, G., Seifert, R. W., , And Hausman, W. 2007. Item-level rfid in the retail supply chain. 16, $65-76$.

G.F.KALSDORF, C. 2006. Clinical supply chain simulation. Ph.D. thesis, Karlsruhe University of Applied Sciences.

HedgePeth, W. O. 2007. RFID Metrics. CRC Press.

Hohberger, C. P. And Tsirline, Y. 2009. Design of a 13,56mhz segmented helmholtz coil for rf exposure testing of biologics to simulated rfid readers. Int. J. Radio Frequency Identification Technology and Applications 2, 1/2, 65-92.

IDTECHEX. 2009. http: / /www. idTechEx.com/. Last Access: 06.01.2009.

IMPACT. 2006. Counterfeit medicines: an update on estimates - 15 november 2006. Available via http: //www . who. int/ [accessed April 6, 2009].

Kelton, W. D., Sadowski, R. P., And Sturrock, D. T. 2007. Simulation with Arena, 4th ed. McGrawHill, Inc, New York.

KLEIJNEN, J. 2005. Supply chain simulation tools and techniques: a survey. $1,1$.

Law, A. And Kelton, W. 2000. Simulation Modeling and Analysis. Mc Graw Series in industrial engineering and management science.

LiN, L. 2009. An integrated framework for the development of radio frequency identification technology in the logistics supply chain management. In Computers \& Industrial Engineering, to appear.

MarCELlis-WARIN, N. D. 2003. Les risques hospitaliers. 40.

Miles, S. B., SARMA, S. E., AND Williams, J. R. 2008. RFID Technology and Applications. Cambridge University Press.

Minvielle, E., Sicotte, C., Champagne, F., Contandripoulos, A.-P., Jeantet, M., Préaubert, N., Bourdil, A., AND Richard, C. 2007. Hospital performance: Competing or shared values? 87, 8-19.

REASON, J. Human errors: Models and management.

REKIK, Y. 2006. The impact of the rfid technology in improving performance of inventory systems subject to inaccuracies. Ph.D. thesis, Ecole CENTRALE Paris.

RoYston, G. 1999. Trials versus modelling in appraising screening programmes. In British Medical journal. Vol. 318. 360-361.

SADIQ, M. 1993. A hybrid clustering algorithm for reconfiguration of dynamic order picking systems. Ph.D. thesis, University of Arkansas.

SARAC, A., ABSI, N., AND DAUZÈRE-PÉRÈs, S. 2008. A simulation approach to evaluate the impact of introducing rfid technologies in a three-level supply chain. In Proceedings of the 2008 Winter Simulation Conference, Miami, U.S.A. 2741-2749.

SEPPANEN, M. 2000. Developing industrial strength simulations models using visual basic for applications. In Proceedings of the 32nd Conference on Winter simulation, Orlando, Florida, USA, pp 77-82.

SwEDBERG, C. 2009. Rfid helps st. joseph's keep an eye on cold storage. RFID Journal - Last Access: 06.01.2009.

ACM: Transactions on Modeling and Computer Simulation, Vol. ?, No. ?, ?? 20?? 
Togt, R. V. D., van Lieshout, E., Hensbroek, R., Beinat, E., Binnekade, J., and Bakker, P. 2008. Electromagnetic interferences from radio frequency identification including hazardous incidents in critical care medical equipment. In Journal of American Medical Association. Vol. 299. 2884-2890.

WorldHealthOrganization. 2006. Counterfeit medicines: Fact sheet. Available via http://www.who.int/[accessed April 6, 2009].

Received June 2009; November 2009; accepted Notyet 2009... 
